UNIVERSIDADE DE BRASILIA - UNB

FACULDADE DE CIÊNCIAS DA SAÚDE

NÚCLEO DE ESTUDOS EM EDUCAÇÃO E PROMOÇÃO DA SAÚDE - NESPROM CENTRO DE ESTUDOS AVANÇADOS MULTIDISCIPINAR - CEAM

CURSO DE ESPECIALIZAÇÃO EM EDUCAÇÃO E PROMOÇÃO DA SAÚDE

Viviane Moreira Dias Lazary

Efeitos do consumo da isoflavona na prevenção do câncer

de mama

PROFESSORA MSC. CAROLINA CONCEIÇÃO PRADO

BRASILIA,

2010 
UNIVERSIDADE DE BRASILIA - UNB

FACULDADE DE CIÊNCIAS DA SAÚDE

NÚCLEO DE ESTUDOS EM EDUCAÇÃO E PROMOÇÃO DA SAÚDE - NESPROM

CENTRO DE ESTUDOS AVANÇADOS MULTIDISCIPINAR - CEAM

CURSO DE ESPECIALIZAÇÃO EM EDUCAÇÃO E PROMOÇÃO DA SAÚDE

Viviane Moreira Dias Lazary

\section{Efeitos do consumo da isoflavona na prevenção do câncer de mama}

Trabalho de Conclusão de Curso - TCC Apresentado para a Banca Examinadora do Curso de Educação e Promoção da Saúde da Universidade de Brasília, como exigência parcial da obtenção do grau de especialista em Educação e Promoção da Saúde.

Professora: Msc. Carolina C. Prado

BRASILIA, 


\section{RESUMO}

A importância da dieta na abordagem ao câncer de mama já é reconhecida, entretanto, os componentes alimentares quimiopreventivos necessitam ser mais bem fundamentados. Este artigo pretender elaborar uma revisão bibliográfica sobre os efeitos benéficos do consumo da isoflavona na prevenção do câncer de mama. Desta pesquisa, foram selecionados dez artigos que discutem as possibilidades de proteção contra o desenvolvimento do câncer de mama, proporcionados por isoflavonóides provenientes da soja. As evidências científicas em relação aos efeitos benéficos do consumo das isoflavonas na prevenção do câncer de mama são baseados em estudos experimentais e epidemiológicos que sugerem que uma alimentação rica em isoflavonóides representa um possível fator de proteção contra o desenvolvimento do câncer de mama. No entanto, algumas evidências sugerem que a genisteína favorece a tumorigênise mamária, fazendo-se necessário que mais estudos sejam realizados, tanto em animais, como em humanos para que possibilite a elucidação dos reais mecanismos das isoflavonas na glândula mamária. Diante das controvérsias encontradas, as pesquisas nesta área devem ser estimuladas, uma vez reconhecida a necessidade de esclarecimento sobre os mecanismos de ação das isoflavonas bem como das quantidades de ingestão desta substância.

Palavras chaves: isoflavonas, alimentos funcionais, dieta, neoplasias, câncer de mama 


\begin{abstract}
The importance of the diet in the mammary cancer approach is known, however, the chemiopreventive food components need to be more well-fundamented. This article intends to elaborate a bibliographic review on the benefic effects of the isoflavones consumption in preventing mammary cancer. This research, tem articles that discuss protection possibilities against mammary cancer development were selected, caused by soy isoflavonoids. The cientific evidence related to the benefic effects of isoflavones consumption in preventing mammary cancer are based in experimental and epidemiologic studies that suggest that a isoflavonoids rich diet a possible protection factor against mammary cancer development. However, some evidence suggest that genistein favors mammary tumorigenesis, being necessary that more studies be conducted, as in animals, as in human beings to make possible to elucidate the real isoflavones engine in the mammary gland. Up front the found controversies, the research in this area must be stimulated, once recognized the need for clarifying the action of the isoflavones engine as well as the quantities of ingestion of this substance.
\end{abstract}

Keywords: isoflavones, functional foods, diet, neoplasys (cancer), breast cancer 


\section{SUMARIO}

RESUMO

$\begin{array}{ll}\text { INTRODUÇÃO } & 01\end{array}$

$\begin{array}{ll}\text { OBJETIVOS } & 03\end{array}$

$\begin{array}{ll}\text { Objetivo geral } & 03\end{array}$

$\begin{array}{ll}\text { Objetivos Específicos } & 03\end{array}$

$\begin{array}{ll}\text { A IMPORTÂNCIA DAS ISOFLAVONAS } & 04\end{array}$

$\begin{array}{ll}\text { Câncer de mama } & 05\end{array}$

$\begin{array}{ll}\text { Isoflavonas X Câncer de mama } & 05\end{array}$

$\begin{array}{ll}\text { METODOLOGIA } & 07\end{array}$

$\begin{array}{ll}\text { RESULTADOS E DISCUSSÃO } & 08\end{array}$

$\begin{array}{ll}\text { CONSIDERAÇÕES FINAIS } & 13\end{array}$

REFERÊNCIAS 


\section{Introdução}

Segundo dados da Organização Mundial de Saúde (OMS), a cada ano o câncer atinge pelo menos 9 milhões de pessoas e mata cerca de 5 milhões, sendo atualmente a segunda causa de morte por doenças na maioria dos países, inclusive no Brasil (DIAS apud CUPPARI, 2005).

Na população mundial, os tumores de colo uterino e da mama feminina são os de maior incidência e causa considerável de mortalidade nas regiões brasileiras (PARKIN apud GAROFOLO, 1999). Segundo informações processadas pelos Registros de Câncer de Base Populacional, disponíveis para dezesseis cidades brasileiras, mostram que na década de 90, o câncer de mama foi o mais freqüente no país. As maiores taxas de incidência foram observadas em São Paulo, no Distrito Federal e em Porto Alegre. O número de casos novos de câncer de mama esperados para o Brasil, no ano de 2010, é de 49.240 (INCA, 2010).

O enfoque ao câncer de mama, na abordagem das neoplasias malignas, justifica a realização de inúmeras pesquisas acerca de sua epidemiologia, fatores de risco, etiologia, métodos diagnósticos, abordagem terapêutica e, especialmente sobre os fatores protetores (WHO, 1998).

Acredita-se que cerca de 35\% dos diversos tipos de câncer ocorrem em razão de dietas inadequadas (DOLL e PETO apud GAROFOLO, 1981). A literatura constantemente destaca a importância da intervenção dietética na prevenção da neoplasia mamária. Dietas baseadas no consumo de frutas, vegetais, grãos integrais e outras plantas parecem atuar na prevenção desta patologia, em decorrência de diversos compostos fitoquímicos, nutrientes ou não nutrientes, que são excelentes agentes quimiopreventivos, frequentemente encontrados nesses alimentos (PADILHA e PINHEIRO, 2004). Nesse contexto, tem sido demonstrado que as isoflavonas da soja, especificamente a genisteína e a daidzeína, apresentam efeito anticancerígeno. Estudos epidemiológicos apontam que as mulheres que consomem dietas ricas em soja e seus produtos, a incidência do câncer de mama é menor quando comparada com as mulheres que não consomem esse tipo de dieta (ESTEVES e MONTEIRO, 2001).

Neste ínterim, este estudo se propõe a reunir informações científicas, em uma revisão bibliográfica, sobre os efeitos benéficos do consumo da isoflavona na prevenção do 
câncer de mama, identificando os componentes químicos, o mecanismo de atuação e as recomendações para o consumo desta substância em relação à prevenção do câncer de mama. 


\section{Objetivos}

\section{Objetivo Geral:}

Elaborar uma revisão bibliográfica sobre os efeitos benéficos do consumo da isoflavona na prevenção do câncer de mama.

\section{Objetivos Específicos:}

- Identificar os componentes da isoflavonas que participam da prevenção do câncer de mama;

- Identificar o mecanismo de atuação das isoflavonas em relação ao câncer de mama;

- Verificar as recomendações para o consumo das isoflavonas; 


\section{A importância das Isoflavonas}

As isoflavonas ou isoflavonóides, são pertencentes a classe dos fitoestrógenos e são compostos químicos fenólicos que constituem o principal tipo de polifenol presente na soja e em outras leguminosas (ESTEVES e MONTEIRO, 2001).

A soja é uma rica fonte das isoflavonas daídzeína, genisteína e gliciteína, as quais apresentam-se com várias formas de conjugados glicosídicos, dependendo da extensão, do processamento ou fermentação. Dois terços do total de isoflavonas são de conjugados de genisteína, sendo o restante composto de conjugados de daidzeína e mínimas quantidades de gliciteína. Nos produtos fermentados de soja, a daidzeína aumenta devido a ação de glicosidases bacterianas (ESTEVES e MONTEIRO, 2001).

Estudos epidemiológicos das últimas décadas evidenciam que o consumo regular de isoflavonas de soja apresentam efeitos protetores de doenças crônicas, doenças cardiovasculares, efeitos da menopausa e em alguns tipos de câncer: mama e próstata (ANJO, 2004).

Segundo informações técnicas da ANVISA, o conteúdo de isoflavonas dos alimentos é extremamente variável, sendo recomendado o consumo diário de produtos com alto conteúdo de isoflavona para se obter uma ingesta suficiente (25mg/dia). O perfil de absorção varia com a dieta, sensibilidade individual, componentes genéticos e fases da vida. A estrutura das isoflavonas, o processamento industrial e a composição do produto também influenciam sua absorção no organismo (ANVISA, 2010).

A ANVISA refere ainda que os efeitos das isoflavonas apenas são reconhecidos para o alívio das ondas de calor associadas à menopausa e como auxiliar na redução de colesterol, já que a quantidade e o período de utilização está relacionado com a condição de saúde do indivíduo e as restrições aos grupos populacionais específicos, de maneira que, demais alegações das isoflavonas, relacionadas a câncer, doenças cardiovasculares e reposição hormonal não possuem comprovação científica suficiente para justificar o seu uso. 


\section{Câncer de mama}

A etiologia do câncer de mama é multifatorial, sendo fundamental o papel dos hormônios endógenos e exógenos. Uma série de fatores de risco relacionados com hormônios endógenos têm sido identificados, como idade tardia ao primeiro parto, nuliparidade, menarca precoce e menopausa tardia. Fatores reprodutivos podem contribuir em até 30\% dos casos de câncer de mama. Sabe-se que o sedentarismo, juntamente com a obesidade e os hábitos alimentares inadequados, podem aumentar consideravelmente esse risco (LIMA, LATORRE e COSTA, 2008).

Vários estudos tem investigado o efeito das gorduras dietéticas no risco de câncer de mama. Estudos de caso-controle mostram forte associação entre uma dieta rica em lipídios e as taxas de incidência de câncer de mama na menopausa. (CIBEIRA e GUARAGNA, 2006).

\section{Isoflavonas X Câncer de mama}

“Diversos estudos epidemiológicos sugerem, indiretamente, que o consumo de isoflavonas dietéticas podem proporcionar proteção contra certos tipos de câncer. Embora estes estudos epidemiológicos isoladamente não sejam conclusivos, eles recebem o apoio de numerosos estudos de laboratório, indicando que as isoflavonas exibem um grande número de efeitos biológicos diversos, tanto in vivo quanto in vitro, e podem estar associadas a atividades anti-câncer” (SHILS et. al. , 2003).

A genisteína é absorvida no estômago de forma eficiente, contudo, não são capazes de inibir um câncer de mama estabelecido, porém, via mecanismos quimioterapêuticos, são suficientes para proliferar a regulação de células epiteliais em câncer, exercendo portanto, efeito quimiopreventivo. (BARNES et. al. , 1996)

A ingestão de isoflavonas desde a infância produz maior efeito na redução dos riscos câncer de mama, embora a dieta com soja a partir da fase adulta também apresente seu efeitos benéficos (WU, 2002). Estudos também demonstram o efeito positivo do uso de isoflavonas como forma de prevenção do câncer de mama em mulheres na fase de prémenopausa. (APPUZIO, 2003) 
Experimentos com indução de câncer de mama e genisteína purificada em modelos animais, têm demonstrado que o tempo de exposição dos animais aos isoflavonóides é crítico. Ratos tratados no período neo-natal ou pré-pubertal com genisteína apresentam tempo de latência maior antes do aparecimento de tumores de mama induzidos e expressiva redução no número de tumores. O mecanismo preventivo da genisteína parece ocorrer pela sua atividade estrogênica que causa diferenciação das células das glândulas mamárias mais rápida. A administração de genisteína após 35 dias de idade apresentou alterações menores, ou seja, redução de 27\% no risco ao câncer de mama. Em animais ovariectomizados a genisteína aumentou a proliferação de células cancerosas comparado com a dieta controle. Futuros estudos sobre o potencial anti-câncer das isoflavonas de soja devem investigar a interação destas com outros componentes fitoquímicos da soja e explorar modelos animais de câncer de mama nos quais, genes específicos podem ser ativados ou inativados (BARNES, 1997). 


\section{Metodologia}

Considerando todos os aspectos destacados e com o objetivo de conhecer os efeitos benéficos do consumo da isoflavona na prevenção do câncer de mama, utilizou-se a metodologia de pesquisa qualitativa que tem por desígnio proporcionar maior proximidade com o problema, com vista a torná-lo mais claro. “O planejamento para este tipo de trabalho é bastante flexível, de modo que possibilite a consideração dos mais variados aspectos relativos ao fato estudado" (GIL, 2002, p. 41).

Tal procedimento metodológico permitiu a avaliação e comparação de dados de pesquisas realizadas recentemente.

Realizou-se uma revisão bibliográfica, utilizando-se o banco de dados da biblioteca virtual Scielo, CAPES, Google Scholars com as palavras chaves: isoflavonas, alimentos funcionais, dieta, neoplasias, câncer de mama. Foram selecionados artigos publicados no idioma inglês e português, que cruzassem pelo menos duas ou mais das palavras chaves relatadas acima.

Foram eleitos artigos originais e de revisão que apresentavam dados entre a relação dos isoflavonóides e a prevenção de neoplasias. Como critério de inclusão, foi definido a necessidade de os artigos possuírem o período de publicação compreendido entre os anos 2000 a 2009

Para obter as estimativas de câncer de mama para o ano de 2010, foi realizado acesso ao site do Instituto Nacional de Câncer (INCA), www.inca.go.br, do Ministério da Saúde. Outro site consultado foi o endereço eletrônico da Agência Nacional de Vigilância Sanitária - (ANVISA), www.anvisa.gov.br, que traz informações técnicas a respeito dos isoflavonóides.

As análises obtidas foram transcritas na forma de texto e tabelas. Tais resultados seguem no próximo capítulo. 


\section{Resultados e Discussão}

Os resultados obtidos estão descritos na tabela 01 .

Tabela 01 - Resultados da revisão bibliográfica (2000-2008).

\begin{tabular}{cc}
\hline Autores & Título \\
\hline $\begin{array}{c}\text { ESTEVES, E.A.; } \\
\text { B. } \text { R. }\end{array}$ & $\begin{array}{c}\text { Efeitos Benéficos das Isoflavonas } \\
\text { de Soja em Doenças Crônicas }\end{array}$ \\
GAROFOLO, & Dieta e câncer: um enfoque \\
A.; et. al. & epidemiológico \\
PADILHA, P. & O Papel dos Alimentos Funcionais \\
C.; PINHEIRO, & na Prevenção e Controle do Câncer \\
R. L & de Mama \\
ROCHA, $M$. C.; & Efeito do Tratamento com \\
OLIVEIRA, J. A. & Isoflavona no Crescimento do \\
V.. & Tumor Sólido de Ehrlich \\
BEDANI, R.; & Isoflavonas: Bioquímica, Fisiologia \\
ROSSI, E. A. & e Implicações para a Saúde
\end{tabular}

PIOVESAN, A. Estudo morfológico e molecular da

C.; et. al. mama de ratas castradas tratadas com isoflavona ou estrogênios

SENA, V. M. G. Efeitos da isoflavona de soja sobre M.; COSTA, L. Os sintomas climatéricos e

O. B. F.; $\quad$ espessura endometrial: ensaio

COSTA, H. L.F clínico, randomizado duplo-cego e .F. controlado
GÓES-FAVONI, Isoflavonas em Produtos
S. .P.; et. al. Comerciais de Soja
Neoplasias, mortalidade, nutrição, epidemiologia, dieta.

Isoflavonas, doenças cardiovasculares, 2001 neoplasias, osteoporose, diabetes mellitus.
Neoplasias mamárias, nutrição, quimioprevenção, alimentos funcionais.

Tumor de Ehrlich; isoflavonas; 2005 fitosetrógenos.

Isoflavonas; proteínas de soja; 2005 osteoporose, câncer; sintomas da menopausa; doenças cardiovasculares.

Mama; estrógenos;isoflavona; 2005 morfologia; ratos.

Menopausa/efeitos de drogas; 2007 isoflavonas/uso terapêutico; fogachos; endométrio/anatomia \& histologia.

Soja; isoflavonas; alimentos à base de 2004 soja; compostos funcionais. 
ROSA, A. M.; $\quad$ Alimentos fermentados à base de et..al.

soja (Glycine Max (Merril) L.): importância econômica, impacto na saúde e efeitos associados às isoflavonas e seus açúcares
Soja; derivados de soja; proteínas;

A soja contém diversos compostos fitoquímicos, alguns dos quais apresentou em estudos epidemiológicos e experimentais, atividade antitumoral, principalmente mama e próstata. Estes efeitos anticancerígenos da soja são associados ao elevado teor de isoflavonas (ROSA; et. al, 2009). No entanto, chama-se a atenção para a existência de diferentes grãos de soja, em que a quantidade e os tipos de isoflavonas são distintos, fato que poderia justificar as divergências encontradas nos diversos estudos (GAROFOLO, A.; et. al, 2004). É importante ressaltar que a biodisponibilidade das isoflavonas na prevenção do câncer de mama apresenta papel fundamental.

As principais isoflavonas verificadas na proteína da soja e nos alimentos a base de soja são: daidzeína, genisteína e gliciteína. Cada uma delas pode ser encontrada em quatro formas: não-conjugadas ou agliconas, conjugada ou glicosilada (daidzina, genistina e glicitina), acetilglicosiladas e malonilglicosiladas (BEDANI; ROSSI, 2005).

A presença e a concentração das isoflavonas nos produtos a base de soja dependem das condições de processamento, principalmente a temperatura de tratamento do material. Produtos não-fermentados têm concentrações de isoflavonas duas a três vezes maiores que produtos fermentados. Alguns alimentos a base de soja também são boas fontes de isoflavonas, como o tofu $(33,7 \mathrm{mg} / 100 \mathrm{~g})$, o miso $(20,4 \mathrm{mg} / 100 \mathrm{~g})$ e o tempeh $(62,5 \mathrm{mg} / 100 \mathrm{~g})$ (GOÉS-FAVONI; et. al, 2004). A ingestão da soja ocorre pelo consumo do próprio grão e através de alimentos elaborados a partir dele. O produto mais amplamente consumido pela população brasileira é o óleo de soja, porém ele não contém isoflavonas. Nesse sentido, uma alternativa interessante para incluir a presença das isoflavonas na alimentação é a criação de produtos enriquecidos com derivados de soja.

Uma importante isoflavona da soja, a genisteína tem atraído muita atenção, não apenas por causa do seu potencial efeito anti estrogênico, mas também porque inibe várias 
enzimas envolvidas em processos de carcinogênese (ESTEVES; MONTEIRO, 2001). A genisteína pode previnir a proliferação mediada por alguns fatores de crescimento através de mecanismos inibidores de tirosina quinase e topoisomerase.

As populações orientais, que apresentam baixa incidência de câncer de mama, consomem de 28-80mg de genisteína por dia, quase toda derivada de produtos de soja, enquanto que a ingestão diária de genisteína nos EUA é somente 1-3mg/dia (ESTEVES; MONTEIRO, 2001). É possível relacionar o aumento no aparecimento do câncer de mama aos hábitos de vida errados, decorrentes de fatores de risco modernos, aos quais a população brasileira também está exposta. O intenso processo de urbanização da população brasileira caracterizado pelo aumento do consumo de alimentos industrializados, redução da atividade física e alterações no comportamento reprodutivo reflete a crescente prevalência da exposição a fatores de risco de câncer de mama.

Aguiar (2002) refere que a genisteína inibiu o crescimento de células cancerosas de mama, regulou a expressão de genes relacionados à apoptose e também reprimiu a proliferação de células cancerosas humanas. Verifica-se portanto que a genisteína além do potencial de inibição do crescimento tumoral pelo fenômeno apoptótico, é capaz de conter a angiogênese. A maioria dos estudos in vitro com culturas de células de câncer de mama evidenciam que altas concentrações de isoflavonas reduzem a proliferação celular, enquanto baixas concentrações exercem efeito estimulatório sobre a proliferação celular(BEDANI; ROSSI, 2005). Ou seja, o aumento ou a diminuição do risco dessa neoplasia parece estar diretamente ligado a dose de isoflavonas administrada.

Embora os mecanismos pelos quais as isoflavonas não sejam totalmente conhecidos, seus efeitos podem estar relacionados à inibição de enzimas, principalmente a topoisomerase II e as tirosina quinases (ROSA; et. al, 2009; AGUIAR, 2009). Existem duas propriedades da genisteína que contribuem para a atividade antiproliferativa das células tumorais: a inativação das enzimas tirosina-quinase, responsável pela proteção das células contra o fenômeno apoptótico e topoisomerase II, essencial à replicação de células cancerosas mamárias. Em adição, outros estudos têm demonstrado propriedades anti-carcinogênicas , antioxidativas, efeitos anti-estrogênicos e anti-proliferativos das isoflavonas (ESTEVES; MONTEIRO, 2001). A propriedade estrogênica das isoflavonas parece decorrer da interação com os receptores de estrogênio (ROSA; et. al, 2009; AGUIAR, 2009). 
Enquanto alguns autores demonstraram que a genisteína possuiria efeito preventivo para o desenvolvimento do câncer de mama devido suas propriedades antiproliferativas, outros autores mostraram que este fito-hormônio estimularia o crescimento de tumores estrogênio dependentes. Estes afirmam que mulheres com dieta rica em isoflavonas (altas doses) poderiam ter proliferação de lesões pré-malignas, sendo o tempo de exposição fator determinante para este efeito (PIOVESAN; et. al., 2005). Entretanto, outros estudos não comprovaram esta hipótese e nem os resultados obtidos pelo referido estudo sugeriram ação proliferativa desses hormônios no tecido mamário.

O estudo que avaliou os efeitos das isoflavonas sobre a morfologia e morfometria da mama de ratas adultas tratadas por período de 60 dias, concluiu que as isoflavonas não apresentam efeitos significantes na proliferação mamária (número de alvéolos/mm2, volume das células alveolares e número de vasos sanguíneos/mm2) (PIOVESAN et. al. , 2005). Resultados semelhantes foram obtidos por outros autores, que avaliaram macacas cinomólogas ooforectomizadas e submetidas a uma dieta rica em isoflavona de soja por longo prazo. Observou-se que a soja parece não estimular a proliferação mamária (SENA; COSTA; COSTA, 2007).

Em outro estudo que avaliou os efeitos das isoflavonas no crescimento do tumor sólido de Ehrlich, tumor originalmente mamário, transplantado para o tecido subcutâneo, constatou-se que a isoflavona é capaz de interferir no ciclo de divisão celular, pois o número de figuras de mitose foi significativamente reduzido no grupo tratado, confirmando assim a capacidade inibitória do crescimento tumoral por sua propriedade antimitótica. O peso e a massa tumoral não apresentaram diferenças entre os grupos, sendo necessário investigação futura (ROCHA; OLIVEIRA, 2005).

Contrariando os achados acima, um estudo que avaliou os efeitos estrogênicos da genisteína no crescimento de células mamárias cancerígenas, tanto in vitro quanto in vivo, resultou na proliferação das células malignas e no aumento dos tumores estrogênio dependentes (PADILHA; PINHEIRO, 2004). Diferente dos outros estudos, a exposição acima sugere um mecanismo de ação dose dependente da relação estrogênica, fato não confirmado pela maioria dos estudos analisados. 
Todos esses estudos são apenas alguns dos diversos já realizados. É possível observar que esses resultados são insuficientes para permitir conclusões definitivas em relação ao uso dos isoflavonóides para a prevenção e/ou tratamento do câncer de mama.

Diante das exposições acima, é necessário avaliar o efeito do consumo de alimentos fontes de isoflavonas como terapêutica em virtude de seus possíveis efeitos adversos. Além disso, a ANVISA não permite a utilização de suplementação com isoflavonas para fins quimiopreventivos. 


\section{Considerações finais}

A relação dos fatores dietéticos com o câncer de mama são constantemente reconhecidos pela literatura, embora não se encontrem totalmente esclarecidos. Nesse sentido, têm sido amplamente estudado a quimioprevenção através do consumo das isoflavonas na prevenção e controle do câncer de mama.

Os estudos analisados referem que os componentes das isoflavonas que atuam na prevenção do câncer de mama são a genisteína e a daidzeína, sendo o primeiro o mais citado. O real mecanismo pelo qual as isoflavonas influenciam este tecido ainda é controverso, sendo atribuídos mecanismos estrogênicos e anti estrogênicos. Outros possíveis mecanismos anticarcinogênicos das isoflavonas estão relacionados à inibição das topoisomerases e tirosina quinases, regulação da progressão, diferenciação e apoptose do ciclo celular.

Diante de algumas evidências que sugerem que a genisteína favorece a tumorigênise mamária, surge a necessidade de que mais estudos sejam realizados, tanto em animais, como em humanos para que possibilite a elucidação dos reais mecanismos das isoflavonas na glândula mamária. Além disso, é importante que haja uma definição das quantidades de ingestão, considerando-se que a ANVISA não reconhece alegações acerca dos efeitos benéficos do consumo das isoflavonas na prevenção do câncer de mama. 


\section{Referências}

AGUIAR, Cláudio Lima. Isoflavonas de soja e propriedades biológicas. B. CEPPA, Curitiba v. 20, n. 2, p. 323-334, jul./dez 2002.

AGUIAR, C.L; CLAVISO, J; PASSOS, L.M.L; ROSA, A.M. Alimentos fermentados à base de soja (Glycine max (Merrill) L.): importância econômica, impacto na saúde e efeitos associados às isoflavonas e seus açúcares. Rev. Bras. Bioci., Porto Alegre, v. 7, n. 4, p. 454462, out/dez. 2009.

AGÊNCIA NACIONAL DE VIGILÂNCIA SANITÁRIA - ANVISA. Perguntas freqüentes sobre alimentos. 2010. Disponível em <http://portal.anvisa.gov.br/Perguntas_Frequentes _Alimentos>. Acesso em 15/01/2010.

ANJO, D.L.C. Alimentos funcionais em angiologia e cirurgia vascular. Jornal Vascular Brasileiro , v.3,n.2, p.145-154,2004.

APPUZIO, D.M. Isoflavones may help prevent premonapausal ER-positive breast câncer. Physician Assistant, v.27, n.1, p.35-38, 2003.

BARNES, S; SFAKIANOS, J; COWARD, L. Soy isoflavonoids and cancer prevention. Underlying biochemical and pharmacological issues. Advances in Experimental Medicine and Biology, New York, v.401, p.87-100, 1996.

BARNES, S. The chemopreventive properties of soy isoflavonoids in animal models of breast cancer. Breast Cancer Research and Treatment., The Hague, v.46, n.2-3, p.169-179, 1997.

CIBEIRA, G.H; GUARAGNA, R.M. Fator de risco e prevenção do câncer de mama. Rev. Nutr., 2006.

CUPPARI, L. Nutrição Clinica no Adulto. 2ª edição; São Paulo: Editora Manole, p. 243-244, 2005.

ESTEVES, E.A; MONTEIRO, J.B.R. Efeitos benéficos das isoflavonas em doenças crônicas . Rev. Nutr., Campinas, v.14, n.1, abr.2001.

GAROFOlO, A.; et. al. Dieta e câncer: um enfoque epidemiológico. Rev. Nutr., Campinas, v.17, n.4, dez. 2004.

GIL, Antônio Carlos. Como Elaborar Um Projeto de Pesquisa. 4. ed. - São Paulo: Atlas. 2002. p. 41- 42.

GÓES-FAVONI, Silvana Pedroso de; et. al. Isoflavonas em produtos comerciais de soja. Ciênc. Tecnol. Aliment., Dez 2004, vol.24, no.4, p.582-586. ISSN 0101-2061. 
INSTITUTO NACIONAL DE CÂNCER. Estimativas 2010: incidência de câncer de mama no Brasil. 2010. Disponível em: <http://www2.inca.gov.br/wps/wcm/connect/tiposdecancer/site/ home/mama>. Acesso em: 12/01/2010.

LIMA, F; LATORRE, M.R.D; COSTA, M.J.C; et. al. Dieta e Câncer no Nordeste do Brasil: avaliação da relação entre alimentação e consumo de grupos de alimentos e câncer de mama. Cad. Saúde Pública, 2008.

PADILHA, P.C.; PINHEIRO, R.L. O Papel dos Alimentos Funcionais na Prevenção e Controle do Câncer de Mama. Revista Brasileira de Cancerologia, 2004; 50(3): 251-260.

PIOVESAN, A.C; et. al., Estudo morfológico e molecular da mama de ratas castradas tratadas com isoflavona ou estrogênio. Rev. Bras. Ginecol. Obstet., Abr 2005, vol.27, no.4, p.204-209. ISSN 0100-7203.

ROCHA, Michelle Corrêa da; OLIVEIRA, João Alexandre Valentim de. Efeito do tratamento com Isoflavona no crescimento do Tumor Sólido de Ehrlich. Salusvita, Bauru, v. 24, n. 3, p. 393-401, 2005.

SENA, V.M.G.M.; COSTA, L.O.B.F; COSTA, H.L.F.F. Efeitos da isoflavona de soja sobre os sintomas climatéricos e espessura endometrial: ensaio clínico, randomizado duplo-cego e controlado. Rev. Bras. Ginecol. Obstet. [online], 2007, vol.29, n.10, pp. 532-537. ISSN 01007203.

SHILS, E.M; OLSON, A.J; SHIKE, M; ROSS, C.A. Tratado de Nutrição Moderna na Saúde e na Doença. 9ª edição; São Paulo: Editora Manole, p. 1363-1370, 2003.

WORLD HEALTH ORGANIZATION. The World Health Report 1998: Life in the 21 st century a vision for all. Geneva: WHO, 1998.

WU, A. Adolescent and adult soy intake and risk of breast cancer in asian-americans. Carcinogenesis, v. 23, n.9, p.1491-1496,2002). 